\title{
Die Erzeugung atomaren Wasserstoffs in $\gamma$-bestrahlten Bakteriophagen und in deren DNS
}

\author{
A. Müller und H. Dertinger \\ Institut für Strahlenbiologie, Kernforschungszentrum Karlsruhe
}

(Z. Naturforsch. 23 b, $83-89$ [1968]; eingegangen am 30. Mai 1967)

\begin{abstract}
ESR-spectra of purified preparations of bacteriophage and of DNA extracted from these objects were found to contain the characteristic lines of atomic hydrogen after $\gamma$-irradiation at $77^{\circ} \mathrm{K}$. The experimental results demonstrate the production of atomic hydrogen by irradiation of organic materials in a direct way. The high stability of the trapped hydrogen atoms is indicated by the fact that heating to $200^{\circ} \mathrm{K}$ for $10 \mathrm{~min}$ reduces the spectral amplitudes by a factor of two only. The yield of trapped atomic hydrogen varied between 0.05 and 0.6 while the total radical yield derived from the ESR-spectra was found between 1.0 and 3.0. Addition of moisture to vacuumdried samples did not enhance the yield of $\mathrm{H}$-atoms. At $50 \%$ water content the yield was also reduced to about $50 \%$ other parameters remaining unchanged. The disappearance of hydrogen lines on heating is accompanied by an enhancement of the octet spectrum due to the addition of atomic hydrogen to thymine groups. The observation of H-stabilization in inorganic phosphates is taken to suggest trapping by phosphate groups in DNA. The efficiency of trapping is probably influenced by the presence of impurities, notably transition metals. The apparent $g$-factor of $2.014 \pm 0.002$ is explained by IS-coupling. The true $g$-factor obtained by application of the B r e i t - R a b i formula is equal to the free electron value within experimental limits of error.
\end{abstract}

Im Verlauf von Arbeiten über primäre Prozesse bei der Einwirkung ionisierender Strahlen auf Bakteriophagen wurde schon früher die Radikalerzeugung in diesen Objekten und in daraus extrahierter Desoxyribonucleinsäure (DNS) mittels der Elektronen-Spin-Resonanz(ESR)-Spektrometrie untersucht ${ }^{1}$. Dabei zeigte sich eine Sättigung der Radikalkonzentration bei hohen Strahlendosen. Dieses Phänomen, das nicht nur bei der DNS, sondern auch bei deren Bausteinen wie Nucleotiden, Nucleosiden, Basen und Desoxyribose auftrat, wurde durch Rekombination von Wasserstoffatomen mit anderen strahlenerzeugten Radikalen erklärt ${ }^{2}$.

Wenn atomarer Wasserstoff in der bestrahlten Materie eingefangen wird und somit eine genügend hohe Lebensdauer erhält, läßt er sich durch sein charakteristisches ESR-Spektrum leicht und eindeutig nachweisen, wie erstmalig von Livingston, ZELDES und TAYLOR gezeigt wurde ${ }^{3}$. Während diese Autoren wäßrige Lösungen anorganischer Säuren nach $\gamma$-Bestrahlung bei $77^{\circ} \mathrm{K}$ untersuchten, fanden später Patten und Gordy H-Atome auch in $\gamma$-bestrahlten wäßrigen Nucleinsäuren bei $4,2{ }^{\circ} \mathrm{K}{ }^{4}$.

Wasserstoffatome verursachen gemäß einer Hypothese von BraAms einen erheblichen Teil des biologi-

1 A. Müller, Int. J. Radiat. Biol. 6, 137 [1963].

2 A. Müller, Akad. Wiss. Lit. Mainz, Abh. math.-naturwiss. Klasse [1964].

3 R. Livingston, H. Zeldes u. E. H. Thylor, Physic. Rev. 94, 725 [1954]. schen Strahlenschadens ${ }^{5}$. Es lag daher nahe, der Entstehung und den weiteren Reaktionen dieser Atome in einem vermehrungsfähigen Objekt, nämlich den Bakteriophagen, mittels der ESR-Spektroskopie nachzugehen. Tatsächlich konnten wir auf diese Weise die Entstehung atomaren Wasserstoffs in organischem Material bei Temperaturen über $77^{\circ} \mathrm{K}$ direkt nachweisen, während die Erzeugung von H-Atomen bisher gewöhnlich nur indirekt aus der Bildung molekularen Wasserstoffs gefolgert wurde. Unsere Untersuchungen der Abhängigkeit der Erzeugung und Stabilität atomaren Wasserstoffs von Temperatur, Wassergehalt und Ausgangsmaterial werden im folgenden dargelegt.

\section{Methodik}

Präparation von Bakteriophagen und DNS: Als Ausgangsmaterial für Bestrahlung und ESR-Messung dienten hochgereinigte Bakteriophagen der Stämme $E$. coli $\mathrm{T} 1$, T2 und T4 $\mathrm{Bo}^{\mathrm{r}}$.Die Herstellung dieses Materials erfolgte nach früher beschriebenen Methoden ${ }^{1}$, die durch Ultrazentrifugation im CsCl-Dichtegradienten und anschließende Dialyse gegen dest. Wasser ergänzt wurden. Die Darstellung der DNS aus den Bakteriophagen folgte gleichfalls den früher angegebenen Verfahren ${ }^{1}$. Nach der abschließenden Gefriertrocknung

${ }^{4}$ R. A. Patten u. W. Gordy, Nature [London] 201, 361 [1964].

5 R. BraAms, Nature [London] 200, 752 [1963]. 
stellten wir die Proben in evakuierten Exsikkatoren über verdünnter $\mathrm{H}_{2} \mathrm{SO}_{4}$ auf bestimmte Wassergehalte ein, die durch Wägung der Proben ermittelt wurden. Anschließend preßten wir die gewonnene Substanz in Tabletten von $3,5 \mathrm{~mm}$ Durchmesser und bis zu $10 \mathrm{~mm}$ Länge wie schon früher beschrieben ${ }^{6}$ und überführten diese in Quarzröhrchen von wenig mehr als $3,5 \mathrm{~mm}$ Innendurchmesser und etwa $10 \mathrm{~cm}$ Länge. Das Evakuieren und Abschmelzen dieser Röhrchen erfolgte, während die darin eingeschmolzenen Proben mit flüssigem Stickstoff tiefgekühlt wurden. Die trockenen Proben wurden vor und nach Herstellung der Tabletten, die ebenfalls unter Vakuum durchgeführt wurde, bis zum Erreichen eines konstanten Gewichts bei mindestens $10^{-5}$ Torr evakuiert.

Bestrahlung: Während der Bestrahlung in einer ${ }^{60}$ Co-Quelle (Gammacell 220, Atomic Energy of Canada) mit einer Dosisleistung von etwa $2 \mathrm{Mrad} / \mathrm{h}$ befanden sich die Proben in einem mit flüssigem Stickstoff gefüllten D e w a r-Gefäß. Anschließend wurde das zur Messung benutzte Ende der Röhrchen mit einem Bunsenbrenner ausgeheizt, um die im Quarz erzeugten, störenden Absorptionszentren zu beseitigen. Währenddessen blieb das andere Ende, das die Probe enthielt, unter flüssigem Stickstoff. Nach dem Eintau- chen und Abkühlen des gesamten Röhrchens ließ man die Tabletten in das ausgeheizte Ende gleiten und überführte es in den vorgekühlten Hohlraum des Spektrometers.

ESR-Spektrometrie: Das für die Messungen benutzte ESR-Spektrometer arbeitete im X-Band bei $9,3 \mathrm{GHz}$ und war mit einem Doppelhohlraum ${ }^{7}$ ausgerüstet. Zur Aufnahme und Wärmeisolierung der Probe während der Messung diente ein doppelwandiges, evakuiertes Quarzrohr von 11mm Außendurchmesser. Dieses Rohr wurde von gasförmigem Stickstoff durchströmt, der vorher durch eine in flüssigen Stickstoff eingetauchte Kupferspirale abgekühlt und daran anschließend nach Bedarf durch eine regelbare elektrische Heizung erwärmt wurde, so daß die Probe auf jede gewünschte Temperatur zwischen $100^{\circ} \mathrm{K}$ und $600^{\circ} \mathrm{K}$ gebracht werden konnte. Als Vergleichsprobe für quantitative Bestimmungen diente ein Kohlepräparat mit $1,15 \cdot 10^{17}$ Spinäquivalenten pro $\mathrm{g}$, das nach früher beschriebenen Methoden geeicht worden war ${ }^{8}$. Zur Auswertung der ESR-Spektren wurde ein Momentenplanimeter verwen$\operatorname{det}^{2}$. Einige vergleichende Experimente der Wasserstofflinien in bestrahlten Zähnen und in anorganischen Phosphaten wurden im Q-Band bei $35 \mathrm{GHz}$ durchgeführt.

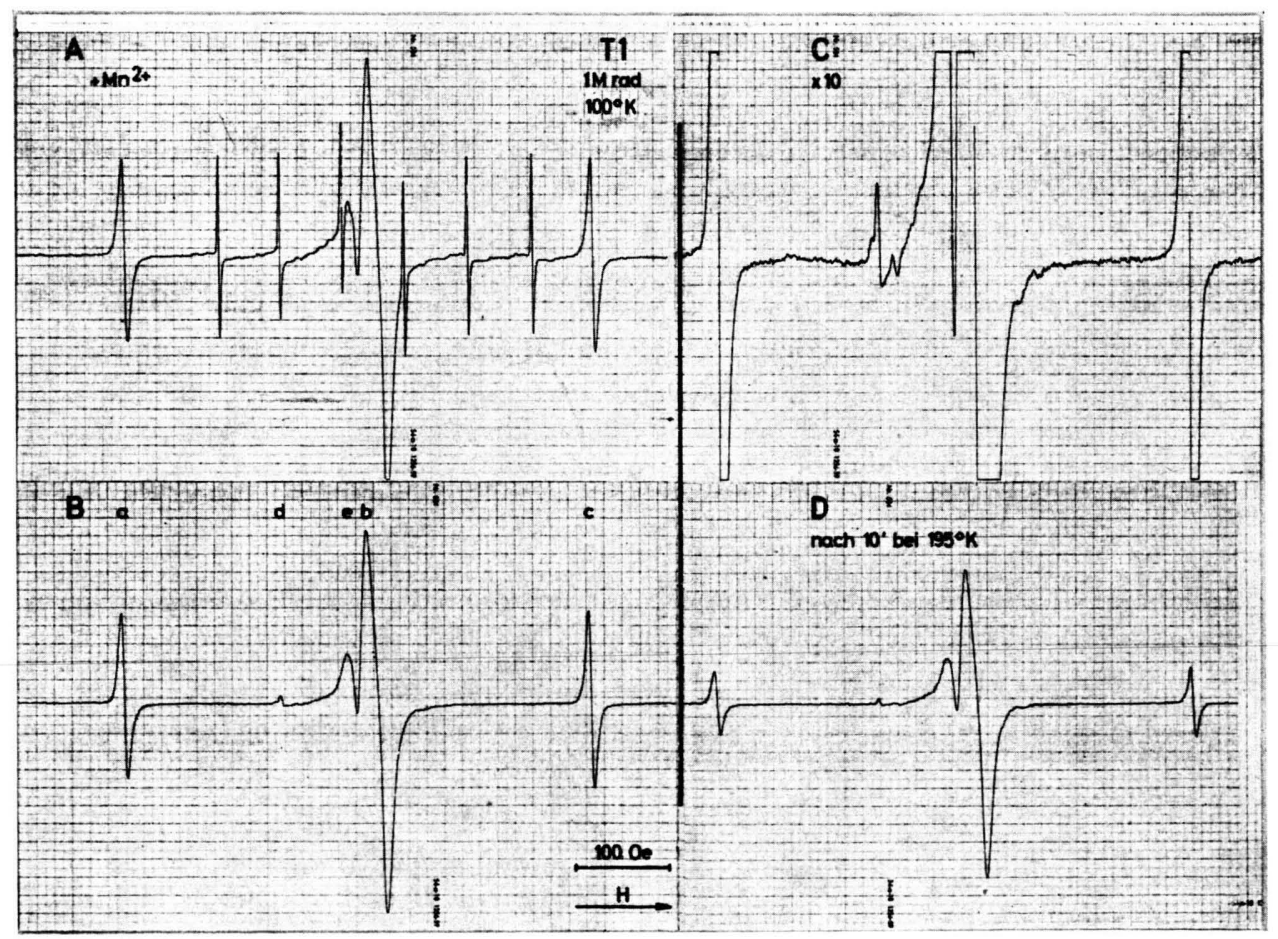

Abb. 1. ESR-Spektren trockener T1-Phagen bestrahlt mit $1 \mathrm{Mrad}$ in vacuo unter flüssigem Stickstoff gemessen bei A) $110^{\circ} \mathrm{K}$ mit $\mathrm{Mn}^{2 \oplus}$ in $\left.\left.\mathrm{ZnS}, \mathrm{B}\right) 130^{\circ} \mathrm{K}, \mathrm{C}\right) 120^{\circ} \mathrm{K}$ und 10 -mal höherer Verstärkung als $\left.\mathrm{B}, \mathrm{D}\right) 120^{\circ} \mathrm{K}$ nach $10 \mathrm{Min}$. Temperung bei $195^{\circ} \mathrm{K}$.

6 A. Müller, G. Hotz u. K. G. Zimmer, Z. Naturforschg. 16 b, 658 [1961].
7 W. Köhnlein u. A. Müller, Z. Naturforschg. 15 b, 138 [1960].

8 W. KöhnLeIN, Strahlentherapie 122, 437 [1963]. 


\section{Ergebnisse}

Gemessene erste Ableitungen der ESR-Absorption von $\gamma$-bestrahlten trockenen T1-Phagen bei $9,3 \mathrm{GHz}$ sind in Abb. 1 wiedergegeben. Während Abb. 1 B nur das Spektrum der Phagen nach Bestrahlung mit 1 Mrad unter flüssigem Stickstoff zeigt, wurde das gleiche Spektrum in Abb. 1 A zur Eichung des Magnetfeldes zusammen mit dem Spektrum von $\mathrm{Mn}^{2 \oplus}$. dotiertem $\mathrm{ZnS}$ aufgenommen. Die beiden Linien a und $c$ sind die für atomaren Wasserstoff charakteristischen. Aus dem Abstand der äußeren beiden der sechs äquidistanten Manganlinien von 340 Oe folgt ein Abstand a - c von $506 \pm 2$ Oe. Der im Vergleich zu dem Manganspektrum niedrigere Mittelwert des Magnetfeldes der H-Linien ist in der Abbildung deutlich zu erkennen. Der daraus folgende $g$-Faktor für das H-Atom beträgt $2,014 \pm 0,001$.

Im Zentrum des Spektrums erscheint die etwa 20 Oe breite Linie b, die wahrscheinlich aus mehreren Linien besteht, mit etwa dem $g$-Faktor des freien Elektrons. Dieser Linie überlagert ist eine andere, relativ scharfe Linie e mit einem $g$-Faktor 2,012 $\pm 0,002$. Schließlich tritt noch eine schwache Linie d mit dem g-Faktor 2,060 $\pm 0,005$ auf, die bei hoher Verstärkung (Abb. 1 C) deutlich zu sehen ist und eine ausgeprägte Struktur zeigt. In Abb. I D ist das Spektrum der gleichen Probe nach 10 Min. dauernder Erwärmung auf $195{ }^{\circ} \mathrm{K}$ in einem AcetonTrockeneis-Bad und anschließender Wiederabkühlung auf $100^{\circ} \mathrm{K}$ dargestellt. Es ist zu erkennen, daß die Intensität der Linien a, c, d und e merklich kleiner geworden, jedoch keinesfalls völlig verschwunden ist.

Die Ausbeute an Wasserstoffradikalen bei $100^{\circ} \mathrm{K}$, die aus den in Abb.1 wiedergegebenen Spektren ermittelt wurde, beträgt $G_{\mathrm{H}}=0,6$, die Ausbeute an anderen Radikalen $G=3,0$. Während $G_{\mathrm{H}}$ und $G$ bei verschiedenen Proben aus dem gleichen Präparat nur eine Streuung von $\pm 20 \%$ zeigen, wie sie bei Absolutbestimmungen der Radikalkonzentration üblich ist, variieren diese Werte bei verschiedenen Präparaten des gleichen Bakteriophagen erheblich stärker. Solche Schwankungen zeigen sich auch bei verschiedenen Präparaten der Phagen T2 und T4 $\mathrm{Bo}^{\mathrm{r}}$ sowie bei Phagen-DNS. Da die Abweichungen zwi-

9 R. Salovey, R. G. Shulman u. W. M. Walsh, JR., J. chem. Physics 30, 839 [1963].

10 A. Ehrenberg, L. Ehrenberg u. G. Löfroth, Nature [London] 200, 376 [1963]. schen verschiedenen Präparaten anscheinend systematischer Natur sind, können über ihre Größe nur ungefähre Angaben gemacht werden. Für die gesamte Radikalausbeute bei tiefer Temperatur wurden $G$-Werte zwischen 1,0 und 3,0 bestimmt, während $G_{\mathrm{H}}$ zwischen 0,05 und 0,6 betrug. Nach den bisherigen Beobachtungen ist die Ausbeute an Wasserstoffatomen bei $\mathrm{T} 2$ und $\mathrm{T} 4 \mathrm{Bo}^{\mathrm{r}}$ sowie daraus gewonnener DNS geringer als bei $\mathrm{T} 1$, jedoch konnte dieser Unterschied wegen der erwähnten Abhängigkeit der Ausbeuten von der Präparation nicht gesichert werden. An ganzen T2-Phagen wurde die Abhängigkeit vom Wassergehalt der Probe untersucht. Das Verhältnis $G_{\mathrm{H}} / G$ war bei Proben mit 45 und $60 \%$ Wassergehalt etwa nur halb so groß wie bei Proben mit einem Wassergehalt unter 20 Prozent. Da für diesen Versuch aliquote Teile des gleichen Präparats, die auf verschiedene Wassergehalte eingestellt waren, verwendet wurden, ist dieses Ergebnis von Unterschieden in der Herstellung unabhängig.

Abb. 2 zeigt die ESR-Spektren einer Probe trockener T2-DNS, bei der die Linien a, c, d und e zwar vorhanden, jedoch erheblich kleiner sind als bei Abb. 1. Dieser Unterschied ist ähnlich dem zwischen T2 und T1 gefundenen, so daß nicht etwa die Proteinfreiheit des Präparats die Ursache der Differenz sein muß. In den Abbn. $2 \mathrm{~B}$ bis $2 \mathrm{E}$ ist die Veränderung des Spektrums bei Erwärmung der Probe wiedergegeben und in Abb. $2 \mathrm{~F}$ das nach Wiederabkühlung gefundene Spektrum. Offensichtlich sind die Linien a, c und d hier vollkommen verschwunden, während der von Linie e scheinbar zurückbleibende Rest auf einer anderen Liniengruppe beruht, wie aus dem folgenden hervorgeht.

Abb. 3 zeigt die ESR-Spektren der gleichen Proben nach Erwärmung auf Zimmertemperatur in anderen Maßstäben als die Abbn. 1 und 2. Die Wasserstofflinien sind unter diesen Bedingungen völlig abwesend. In allen Spektren, besonders aber in dem mit B bezeichneten, sind Satelliten geringer Intensität zu erkennen, die für bestrahltes Thymidin charakteristisch sind und schon früher in bestrahlter DNS gefunden wurden ${ }^{9,10}$. Die Struktur des zugehörigen Radikals wurde von Eisinger und Shulman mit $\mathrm{R}_{1} \mathrm{CH}_{2} \mathrm{C}^{*} \mathrm{R}_{2} \mathrm{CH}_{3}$ angegeben ${ }^{11}$. Die Konzentration dieser Radikale betrug nach Bestrahlung bei

11 J. Eisinger u. R. G. Shulman, Proc. nat. Acad. Sci. USA 50, 694 [1963]. 


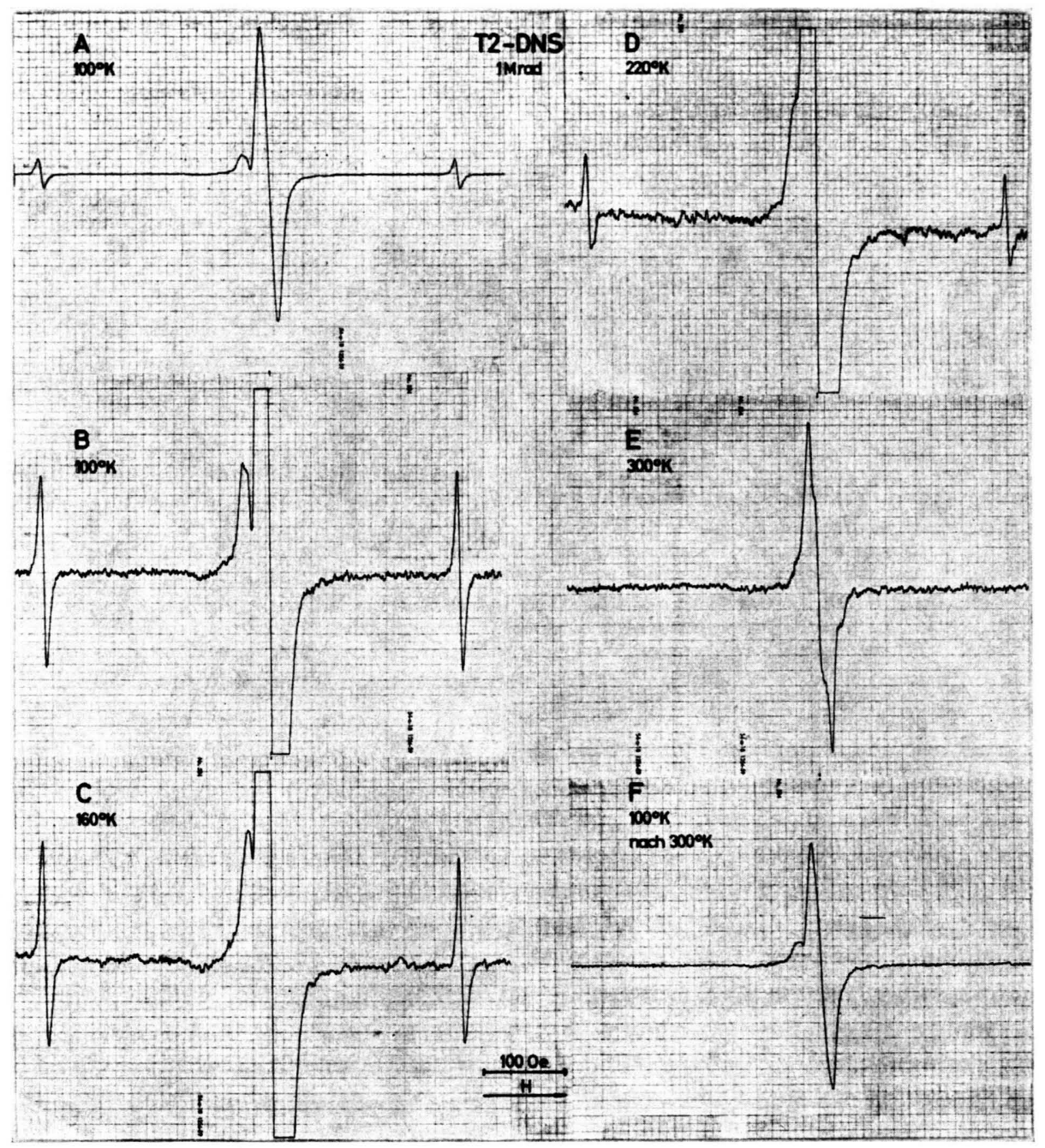

Abb. 2. ESR-Spektren trockener T2-DNS bestrahlt mit $1 \mathrm{Mrad}$ in vacuo unter flüssigem Stickstoff gemessen bei A) $100{ }^{\circ} \mathrm{K}$, B) $95^{\circ} \mathrm{K}$ und 7-mal höherer Verstärkung als A, C) $160^{\circ} \mathrm{K}$, D) $220^{\circ} \mathrm{K}$, E) $\left.300^{\circ} \mathrm{K}, \mathrm{F}\right) 100^{\circ} \mathrm{K}$ nach Erwärmung auf $300{ }^{\circ} \mathrm{K}$.

$77^{\circ} \mathrm{K}$ und Erwärmung auf $300^{\circ} \mathrm{K}$ bis zu $25 \%$ der gesamten Radikalkonzentration. Dieser Anteil war auch nicht wesentlich höher bei Proben, die bis zu 60\% Wasser enthielten. Ein Vergleich mit Abb. 2 zeigt, daß dem Anteil der Linie e, der bei Temperaturerhöhung zusammen mit den Wasserstofflinien verschwindet, eine Oktettlinie überlagert ist, die bei Zimmertemperatur an Stelle der Linie e übrig bleibt. Weiterhin zeigt ein Vergleich der Abb. $1 \mathrm{C}$ mit $3 \mathrm{~B}$ sowie $2 \mathrm{~A}$ mit $3 \mathrm{C}$, daß die Oktettlinien zwar schon bei tiefer Temperatur und hoher Verstärkung andeutungsweise zu erkennen sind, daß sie jedoch nach Erwärmung auf $300{ }^{\circ} \mathrm{K}$ und dem Verschwinden der $\mathrm{H}$-Linien stärker hervortreten. Innerhalb der Meß- genauigkeit besteht auch quantitative Utbereinstimmung zwischen beiden Erscheinungen, doch ist der mögliche Fehler bei der Auswertung des Oktettspektrums der Konzentration der $\mathrm{H}$-Atome angenähert gleich.

Außerdem zeigt Abb. 3 die Spektren beider Proben in einem zehnfach größeren Bereich des Magnetfeldes. Die Absorptionslinien der strahlenerzeugten Radikale bei 3320 Oe erscheinen diesem Maßstab entsprechend schmal. Zwischen der niedrigsten dargestellten Feldstärke von etwa $0 \mathrm{Oe}$ und den strahlenerzeugten Linien ist eine langsam veränderliche Absorption zu erkennen, wie sie schon früher in Bakteriophagen und deren DNS gefunden ${ }^{6}$ und den 
in diesen Präparaten als Spuren enthaltenen paramagnetischen Metallen zugeschrieben wurde ${ }^{6,12}$.

Es ist nicht ohne weiteres möglich, aus der gefundenen Absorption den Gehalt an diesen Metallen zu ermitteln, da einmal deren Zusammensetzung unbekannt ist und zum zweiten ferromagnetische Aggregate gebildet werden können, deren Absorption wesentlich größer ist als die der isolierten Atome ${ }^{12}$.

\section{Diskussion}

Die dargestellten ESR-Spektren zeigen, daß atomarer Wasserstoff bei der Bestrahlung von Bakterio-

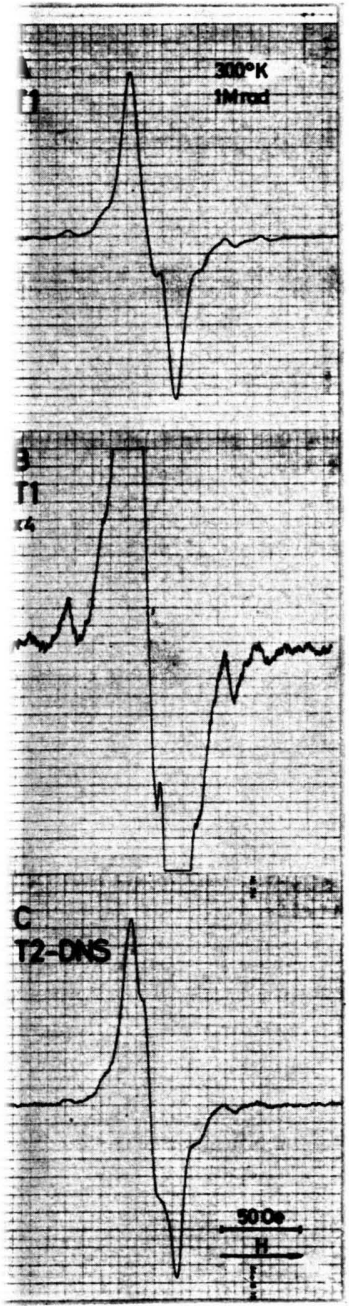

Abb. 3 a.

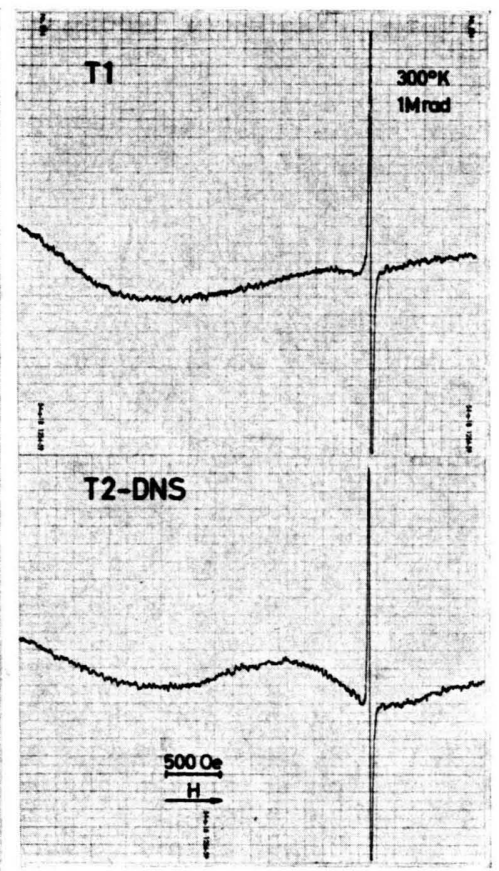

Abb. 3 b.
Abb. 3. ESR-Spektren trockener T1-Phagen und T2-DNS bestrahlt mit 1 Mrad in vacuo unter flüssigem Stickstoff gemessen bei $300{ }^{\circ} \mathrm{K}$ mit verschiedenen Verstärkungen und Bereichen der magnetischen Feldstärke.

12 W. M. Walsh, JR., R. G. Shulman u. R. D. Heidenreich, Nature [London] 192, 1041 [1961].

13 T. Cole u. A. H. Silver, Nature [London] 200, 700 [1963]. phagen und Phagen-DNS gebildet und an bestimmten Stellen gebunden wird. Die Stabilität dieser Bindung ist so groß, daß nach der Entstehung der $\mathrm{H}$ Atome bei $77^{\circ} \mathrm{K}$ die Temperatur der Präparate bis auf über $200^{\circ} \mathrm{K}$ erhöht werden kann, ohne daß die Wasserstofflinien völlig verschwinden. Eine ähnlich starke Bindung atomaren Wasserstoffs ist in der anorganischen Chemie von Phosphaten bekannt. Tatsächlich wurde in $\gamma$-bestrahlten Milchzähnen langlebiger atomarer Wasserstoff mittels seines ESRSpektrums sogar bei Zimmertemperatur von CoLE und Silver nachgewiesen ${ }^{13}$. Ein entsprechendes Ergebnis erhielten wir nicht nur mit verschiedenen Zähnen, sondern auch mit mineralischen Apatiten und anorganischen Calciumphosphaten. Diese Resultate legen die Vermutung nahe, daß die H-Atome in der DNS ebenfalls durch Phosphatgruppen stabilisiert werden. Diese Annahme ist in Ubereinstimmung mit der relativ großen Breite der Wasserstofflinien von etwa 80 e sowie der Abwesenheit von Mikrowellensättigung auch bei relativ hoher absorbierter Leistung.

Wir nehmen an, daß die starken Schwankungen der Ausbeute an H-Atomen nicht durch eine entsprechende Variabilität der Bildung zustande kommt, sondern durch Veränderungen der Anzahl von "trapping sites" oder Einfangzentren für atomaren Wasserstoff. Aus der Festkörperphysik ist bekannt, daß derartige Einfangprozesse gewöhnlich stark von geringen Verunreinigungen abhängen. Es ist weiterhin bekannt, daß die DNS überaus feste Komplexe mit Metallionen bildet ${ }^{12}$ und da $\beta$ die Phosphatgruppen wahrscheinlich an solchen Bindungen beteiligt sind. Eine Beeinflussung der H-Stabilisierung durch DNS-gebundene Metallionen ist daher zu erwarten. Eine beträchtliche Einwirkung auf die Ausbeute an anderen stabilen Radikalen bei Bestrahlung durch die Anwesenheit paramagnetischer Metalle wurde schon früher nachgewiesen ${ }^{\mathbf{1 4}}$.

Im Gegensatz zu Pershan et al., die eine starke Zunahme des Oktettspektrums mit steigendem Feuchtigkeitsgehalt von DNS nach Bestrahlung bei tiefer Temperatur beobachteten ${ }^{14}$, konnten wir keine deutliche Abhängigkeit dieses Anteils vom Wassergehalt der Probe feststellen. Auch bei etwa 50\% Feuchtigkeit blieb dieser Anteil stets unter 25\% der gesam-

14 P. S. Pershan, R. G. Shulman, B. J. Wyluda u. J. Eisinger, Physics 1, 163 [1964]. 
ten Radikalkonzentration. Auch die Ausbeute an stabilisierten $\mathrm{H}$-Atomen zeigte keine solche Abhängigkeit, sondern nahm, wie oben dargelegt, auf die Hälfte ab, wenn der Wassergehalt auf 45 oder $60 \%$ erhöht wurde. Patten und Gondy berichteten eine Zunahme der Wasserstofflinien mit ansteigendem Wassergehalt von DNS ${ }^{4}$. Diese ist wahrscheinlich auf H-Atome zurückzuführen, die mit Hilfe des Wassers stabilisiert werden, da bei der Temperatur von $4,2{ }^{\circ} \mathrm{K}$ auch in reinem bestrahltem Wasser stabile H-Atome gefunden wurden ${ }^{15}$. Die Abwesenheit der Wasserstofflinien nach Bestrahlung wasserfreier DNS, die Patten und Gondy berichteten ${ }^{4}$, wird durch die oben schon diskutierte Variabilität der Einfangbedingungen erklärt.

Im Gegensatz zu früheren Bestimmungen ${ }^{3,4,13,16}$ fanden wir für den scheinbaren $g$-Faktor des $\mathrm{H}$ Spektrums durch Vergleich mit Manganspektren den sehr hohen Wert von 2,014 $\pm 0,002$. Um einen Irrtum auszuschließen, wurden vergleichende Messungen an H-Atomen in bestrahlter Schwefelsäure bei $77^{\circ} \mathrm{K}$ und Vergleiche mit DPPH als Feldmarker durchgeführt. Linearität und Absolutwerte der magnetischen Feldstärke wurden jeweils mittels Protonenresonanz bestimmt. Alle diese Versuche bestätigen lediglich den oben angegebenen Wert, und zwar nicht nur für strahlenerzeugte H-Atome in Bakteriophagen- und DNS-Präparaten, sondern auch in $\mathrm{H}_{2} \mathrm{SO}_{4}$ und $\mathrm{CaHPO}_{4}$. Das Studium der Literatur und eigene Messungen zeigen, daß bei ESR-Messungen im K- oder Q-Band eine weniger große Abweichung vom $g$-Faktor des freien Elektrons gefunden wurde ${ }^{3,4,13}$. Im von uns benutzten X-Band verhalten sich Kern- und Elektronenspin am wenigsten dem P a s c he n - B a c k - Effekt entsprechend unabhängig voneinander, sondern zeigen eine gewisse Kopplung, die durch die Breit-Rabi-Formel zum Ausdruck gebracht wird ${ }^{17}$. Wie im Anhang zu dieser Arbeit gezeigt wird, ergibt die Größe dieser Verschiebung gerade die gefundene Abweichung des g-Faktors vom Wert des freien Elektrons und damit eine Auflösung des obigen Widerspruchs. Die im XBand auftretende Abweichung des scheinbaren $g$ Faktors vom Wert des freien Elektrons ist auch in den von Henriksen veröffentlichten Spektren zu er-

15 L. H. Piette, R. C. Rempel, H. E. Weaver u. J. M. FlourNox, J. chem. Physics 30, 1623 [1959].

16 C. K. J J , in: Formation and Trapping of Free Radicals (eds. Bass u. Broida), S. 213. Academic Press, New York 1960. kennen, wird jedoch nicht explizit angegeben ${ }^{18}$. Innerhalb der Fehlergrenzen ist der gemessene $g$-Faktor der Linie e der gleiche wie der der Wasserstofflinien. Die geringe Breite dieser Linie von etwa 8 Oe entspricht ebenfalls den beiden Wasserstofflinien. Diese Übereinstimmungen wären erklärlich, wenn die Linie e eine Nebenlinie zweiter Ordnung der Wasserstofflinien wäre. Sie käme dann durch „verbotene" Übergänge zustande, bei denen der Kernspin des Protons gleichzeitig mit dem Spin des an dieses Proton gebundenen Radikalelektrons umklappt. Die Übergangswahrscheinlichkeit dieser ,verbotenen" Linie wird im Anhang ebenfalls theoretisch abgehandelt, doch ergibt sich aus der numerischen Berechnung eine viel kleinere Intensität als die beobachtete. Auch entspricht die Amplitudenänderung der Linie e bei Temperaturerhöhung nicht der der Linien a und c. Somit erscheint die Annahme nicht gerechtfertigt, daß diese Linie allein durch den verbotenen Übergang zustande kommt. Die Linie d mit ihrem noch höheren $g$-Faktor 2,060 kann bisher ebenfalls nicht befriedigend gedeutet werden. HENRIKSEN fand in bestrahlten wäßrigen Lösungen ebenfalls Linien mit $g=2,013$ und 2,056 ${ }^{18}$. Diese Werte stimmen zwar gut mit denen der Linien $\mathrm{d}$ und e überein, doch sind die entsprechenden Linien in den Spektren von Henriksen wesentlich (etwa 5-fach) breiter. Die Linie mit dem $g$-Faktor 2,056 wird von HeNRIKSEN dem solvatisierten $\mathrm{O}^{\ominus}$. Radikal zugeschrieben.

Frl. B. Kammerer, Herrn W. Heger und Herrn D. Alke danken wir für sorgfältige und fleißige technische Assistenz bei der Durchführung der Experimente.

\section{Anhang}

An dieser Stelle soll der Beweis zweier in der vorhergehenden Arbeit ausgesprochener Behauptungen erbracht werden, daß nämlich 1 . der hohe $g$-Faktor des Wasserstoff-Dubletts durch die Mitberücksichtigung feldabhängiger höherer Terme in der Resonanzbedingung des Elektronenspins erklärt werden kann und 2. die Linie e in Abb. 1 aus Intensitätsgründen nicht mit der verbotenen Wasserstofflinie identifiziert werden darf.

Zum Beweis der ersten These verfährt man zweckmäßigerweise folgendermaßen: Ausgehend von der

17 G. Breit u. I. I. Rabi, Physic. Rev. 38, 2082 [1931].

18 T. IIfNRIKsen, Radiat. Res. 23, 63 [1964]. 
Breit-RaBi-Formel ${ }^{17}$ wird die Resonanzfeldstärke $H_{0}{ }^{\prime}$ nach Potenzen der Hyperfeinstruktur-Kopplung $A$ entwickelt ${ }^{19}$ :

$$
H_{0}{ }^{\prime}=H_{0}+A m-\left(A^{2} / 2 H_{0}\right)\left[I(I+1)-m^{2}\right]+\ldots
$$

Darin bedeuten: $H_{0}=h v / g \beta$ die Feldstärke des ungestörten $\mathrm{Z}$ e e m a n - Übergangs $(A=0), A=$ die Hyperfeinstruktur-Kopplung in Einheiten der magnetischen Feldstärke, $I=$ die Kernspin-Quantenzahl, $m$ $=$ die Komponente des Kernspins in Richtung des äußeren Magnetfeldes.

Bei den vorliegenden Größenverhältnissen dürfen höhere Glieder der Entwicklung vernachlässigt werden. Ohne den in $A$ quadratischen Term stellt die obige Gleichung die übliche Form der Resonanzbedingung dar. Die Korrektur bewirkt offenbar eine Verschiebung des ESR-Spektrums zu geringerer Feldstärke, die nur bei großen Werten von $A$ oder auch bei schwachem äußeren Magnetfeld ins Gewicht fällt. Formal wird dieser Effekt durch eine Erhöhung des $g$-Faktors beschrieben. Mit der Bezeichnung

$$
\Delta H=\left(A^{2} / 2 H_{0}\right)\left[I(I+1)-m^{2}\right]
$$

folgt für die Erhöhung $\Delta g$ des $g$-Faktors :

$$
\checkmark g=g \Delta H / H_{0} \text {. }
$$

Nun gilt für das Wasserstoffatom: $I=|m|=1 / 2$. Zusammen mit den im Text angegebenen Werten von $A$ $=506 \mathrm{Oe}$ und $H_{0}=3320 \mathrm{Oe}$ ergibt sich somit für den
g-Faktor $g_{\mathrm{H}}$ des atomaren Wasserstoffs, wenn für $g$ der Wert des freien Elektrons $g=2,0023$ eingesetzt wird:

$$
g_{\mathrm{H}}=g+\Delta g=g+(g / 4)\left(A / H_{0}\right)^{2}=2,0139,
$$

was mit dem experimentell gefundenen Wert von $g_{\mathrm{H}}$ $=2,014$ gut übereinstimmt.

Der Beweis der zweiten These erfordert eine Abschätzung der Intensität des in erster Näherung verbotenen Übergangs $\Delta m= \pm 1$, der jedoch unter dem störenden Einfluß der großen Kernspin-Kopplung $A$ erheblich verstärkt wird. Die relative Amplitude $r$ dieses Übergangs, der genau zwischen den beiden Linien des Wasserstoff-Dubletts liegt, ist als Quotient aus verbotener und erlaubter Übergangswahrscheinlichkeit gegeben durch ${ }^{19}$ :

$$
r=\left(A^{2} / 2 H_{0}^{2}\right)[I(I+1)-m(m+1)] .
$$

Für atomaren Wasserstoff ist $I=1 / 2$ und $m=-1 / 2 \mathrm{zu}$ setzen und somit erhält man mit $A=506 \mathrm{Oe}$ und $H_{0}$ $=3320$ Oe:

$$
r=(1 / 2)\left(A / H_{0}\right)^{2}=1 / 86 .
$$

Die Linie e in Abb. 1 ist hinsichtlich ihrer Amplitude demnach nicht mit der verbotenen Wasserstofflinie identisch.

19 J. S. Hyde, U. Ranon u. W. Landgraf, Varian/Tech. Information $[1965 / 66]$. 